\title{
A Flower Specific Calcineurin B-Like Molecule (CBL)-Interacting Protein Kinase (CIPK) Homolog in Tomato Cultivar Micro-Tom (Solanum lycopersicum L.)
}

\author{
Takashi Yuasa*, Yushi Ishibashi, Mari Iwaya-Inoue \\ Department of Bioresource Science, Faculty of Agriculture, Kyushu University, Fukuoka, Japan. \\ Email: ${ }^{*}$ yuasa@agr.kyushu-u.ac.jp
}

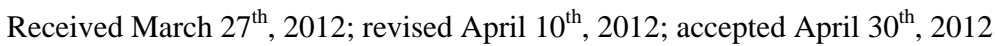

\begin{abstract}
Floral and reproductive organs of higher plants are relatively sensitive to biotic and abiotic stresses compared with the vegetative organs. Calcineurin B-like molecule (CBL) interacting protein kinase (CIPK) has appeared to be involved in acquired tolerance and acclimation under environmental stresses such as salinity, drought and chilling. Semi-quantitative RT-PCR using the vegetative and reproductive organs of tomato Micro-Tom (Solanum lycopersicum L.) at the various developmental stages indicated that SICIPK2 was expressed specifically in the floral organ. An anti-CIPK specific antibody recognized the recombinant SICIPK2 specifically and cross-reacted to a CIPK-related polypeptide at a significant level in flower, particularly in stamen. The flower specific CIPK was tightly associated with the microsomes. In vitro pull-down assay of the recombinant SICIPK2 showed that SICIPK2 interacts with SICBLs and stress-responsive transcription factors, SIERF7, SICBF1 and SIAREB1. The present data suggested that the flower-specific CIPK, SICIPK2, was involved in calcium signaling in tomato via CBLs and stress tolerance possibly mediated by the stressresponsive transcription factors in stamen.
\end{abstract}

Keywords: Calcium; CBL; CIPK; Flower; SnRK; Tomato

\section{Introduction}

In higher plants, the floral development is well programmed and plays an important role in plant reproduction. Compared with the vegetative development, the reproductive development is relatively susceptible to environmental stresses such as high temperature, drought and chilling. For an example of tomato races, the fruit production under moderate heat shock is dependent on that genetic background, but there was marginal difference in neither photosynthetic rate nor respiration between the heat tolerant race and the sensitive race, when subjected to high temperature stress. Reduction of tomato fruit yield under high temperature stress was mostly due to failure of pollen development, such as formation of sterile pollen. A chaperone, HSP90, has appeared to be expressed specifically in floral organs of Arabidopsis and rice in particular, in pollen [1,2]. Pollen accumulates osmolites, proline and sucrose at high concentration, indicating that specific protective mechanisms against heat and/or osmotic stress are involved in development of pollen [3].

In plants, complex mechanisms including stress signaling cascades and synthesis of osmolytes have been evolved

${ }^{*}$ Corresponding author. to survive under various environmental stresses [4]. For molecular and genetic breeding directing to improvement of stress tolerance during reproduction stage of crops, investigation of intracellular mechanisms of stress signaling of floral organs and fruit, which are supposed to be susceptible to various stresses, becomes important in an aspect of agriculture encountering to the situation of global warming.

It is well known that protein phosphorylation/dephosphorylation has an essential role in stress tolerance and adaptation of eukaryotes [5,6]. Recent studies of organ specific SNF1-related protein kinases (SnRKs) revealed that Vicia faba guard cell-specific SnRK2, ABA-activated protein kinase (AAPK) [7], and A. thaliana root tip-specific SnRK2C/SnRK2.8 [8] are involved in closure of guard cells and tolerance of osmotic stress, respectively, and that tomato fruit-specific SnRK2 homolog, SISnRK2C, are activated by $\mathrm{NaCl}$ and chilling [9].

In plant cells, $\mathrm{Ca}^{2+}$ ion is a second messenger involveing the regulation of enzymatic activity and gene expression via calmodulins and $\mathrm{Ca}^{2+}$-dependent protein kinases. Recent studies have clarified that a calcium sensor "Calcineurin B-Like protein” (CBL), containing $\mathrm{Ca}^{2+}$-binding EF-hand motifs, interacts to CBL-Interacting Protein 
Kinases (CIPKs) [10]. The CIPKs contain a kinase domain which has significant similarity in aminoacid sequence to highly similar to SNF1, at the N-terminus and a specific interacting domain, containing a NAF/FISL motif, associating to $\mathrm{CBL}$ at the $\mathrm{COOH}$ regulatory domain $[10,11]$. On the basis of these structural similarities, the CIPKs belongs to the SnRK3 subgroup of plant SNF1-related kinases [12]. Biochemical and genetic research by Arabidopsis mutants have revealed that the CBL-CIPK complex is translocated onto microsomes and implicated in ion homeostasis, solute transport and aquring tolerance under environmental stresses [13,14].

Tomato cultivar Micro-Tom (Solanum lycopersicum L.) is a model crop and suitable for research of fruit, secondary metabolisms and environmental signaling such as pathogenic stress and temperature stresses, etc. We profiled expression levels of CIPK homologs in tomato organs and identified SICIPK2 as a flower specific CIPK. Immunoblot and pull-down assay indicated that the flowers specific CIPK is localized in stamen, associating to the microsomal fraction and interacts with stress-responsive transcription factors, suggesting involvement of pollen development and protection against environmental stresses.

\section{Materials and Methods}

\subsection{Plant Materials}

Seeds of the dwarf tomato (Solanum lycopersicum L. cv. Micro-Tom) were supplied from the Kazusa DNA Research Institute in Japan. Tomato seeds were incubated in 6-cm-square rock wool under continuous light $\left(80 \mathrm{~W} \cdot \mathrm{m}^{-2}\right)$ and fertilized with 1 to 1000-diluted Hyponex (Hyponex Japan, Osaka, Japan). Various organs from 6 - 8 week old tomato plants were frozen in liquid $\mathrm{N}_{2}$ for source of total RNA and protein samples.

\subsection{RNA Extraction and Semiquantitative RT-PCR of Tomato CIPK and CBL Orthologs}

At least, 9 sequences among "contigs" coding tomato CIPK orthologs were predicted by BLAST search using Arabidopsis CIPK1 [10] and CIPK24/SOS2 [15] as search terms against the Micro-Tom EST database (MiBASE) and Kazusa Tomato Unigene ver. 3 (KTU3) (http://www. kazusa.or.jp/jsol/microtom/indexj.html). The deduced amino acid sequences of the tomato CIPK candidates and Arabidopsis CIPKs (http://mips.gsf.de/proj/thal/db/index.html) were aligned by the CLUSTALW program (http://align. genome.jp/). Eventually, we focused on 4 candidates, SICIPK1, 2, 8 and SISOS2, because of putatively higher expression levels of the genes than those of other tomato CIPK orthologs based on numbers of ESTs supporting those contigs in MiBASE. SICIPK1, SICIPK2 (KTU3 contig27053, AB674507, this study) and SICIPK8 were designated corresponding to AtCIPK1, AtCIPK2, AtCIPK8 and AtCIPK24/SOS2, respectively (Figure 1(b)). Similarly, tomato CBL orthologs, SICBL1, 2, 10 and SISOS3, were designated corresponding to AtCBL1, AtCBL2, AtCBL10 and AtCBL4/SOS3 (Figure 2(c)).

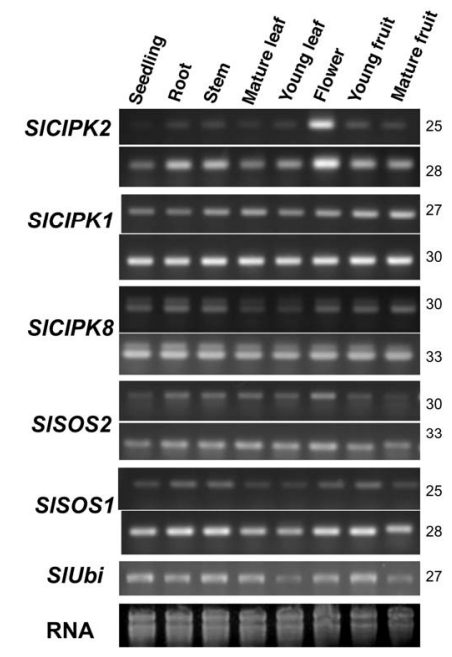

(a)

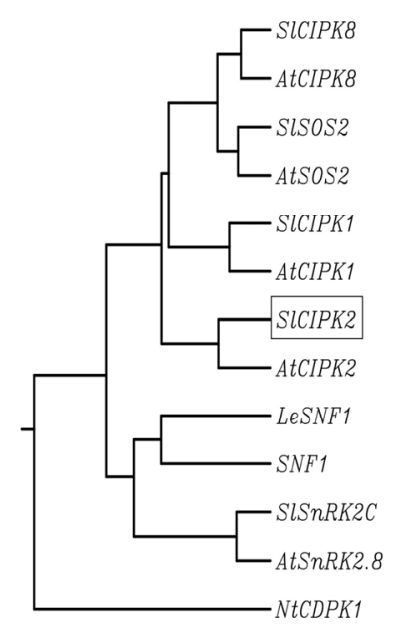

(b)

Figure 1. The expression profiles of tomato CIPK homologs in various organs of tomato plant. (a) The expression levels of mRNAs of SICIPK2, SICIPK1, SICIPK8, SISOS2 and SISOS1 in various organs are shown. Semi-quantitative RT-PCR was carried out at indicated numbers of thermal cycles and with cDNA prepared from various organs and sets of specific primers for detection of tomato CIPK homologs and SISOS1. SIUbi was used as a standard; (b) A phylogenic tree of CIPKs, SNF1-related kinases from tomato and Arabidopsis was aligned by ClustalW (UPGMA). The genes used were follows: SICIPK1, 2,8 and SISOS2 (Table 1); AtCIPK1, At3g17510; AtCIPK2, At5g07070; AtCIPK8; At4g24400; AtSOS2 At5g35410; LeSNF1, AF143743; yeast SNF1, NP010765; SISnRK2C, AB275884; AtSnRK2.8, AF41782; NtCDPK1, AF072908. 


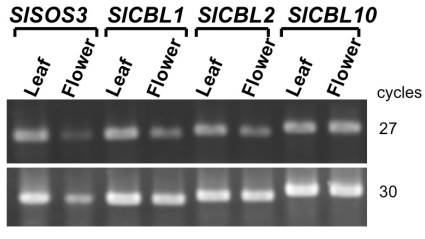

(a)

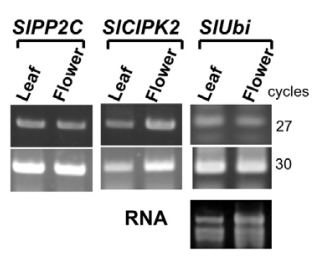

(b)

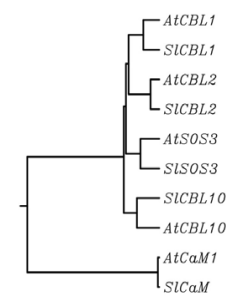

(c)

Figure 2. The expression profiles of tomato CBL homologs, SICIPK2, SIPP2C and SIUbi in tomato leaf and flower. (a) The expression levels of tomato CBLs; (b) The expression levels of SICIPK2, SIPP2C and SIUbi. Semi-quantitative RT-PCR was carried out at indicated numbers of thermal; (c) A phylogenic tree of CBLs from tomato and Arabidopsis was aligned by ClustalW (UPGMA). The genes used were follows: SICBL1, 2, 10 and SISOS3 (Table 1); AtCBL1, At4g17615; AtCBL2, At5g55990; AtCBL10, At4g33000; AtCBL4/SOS3, At5g24270; tomato calmodulin (LeCaM), KTU3 Contig29992; Arabidopsis calmodulin (AtCaM1), At5g37780.

Total RNA was isolated from samples by the SDS/ phenol/LiCl method. For semi-quantitative RT-PCR, cDNA synthesis and PCR were performed with M-MLV reverse transcriptase (TaKaRa, Tokyo, Japan) and GoTaq Green Master Mix (Promega, Tokyo, Japan), respectively, according to the manufacturer's manuals. Each $20 \mu \mathrm{L}$ of PCR reaction mixture contained $10 \mathrm{~mL}$ of $2 \times$ GoTaq mixture, $400 \mathrm{nM}$ each 5'- and 3'-primer and $1 \mu \mathrm{L}$ of tomato cDNA. The thermal cycle reaction was performed as follows: $94^{\circ} \mathrm{C}$ for 2 min; 25 - 30 cycles (as indicated) of $94^{\circ} \mathrm{C}$ for $10 \mathrm{~s}, 58^{\circ} \mathrm{C}$ for $10 \mathrm{~s}$, and $72^{\circ} \mathrm{C}$ for $30 \mathrm{sec} ; 72^{\circ} \mathrm{C}$ for $30 \mathrm{sec}$; and $4^{\circ} \mathrm{C}$ for $99 \mathrm{~min}$. Gene-specific primers for tomato CIPKs, tomato CBL homologs, a tomato putative $\mathrm{Na}^{+} / \mathrm{H}^{+}$ antiporter (SISOS1) ortholog and tomato ubiquitin (SIUbi) are shown in Table 1. The PCR products separated in $1.5 \%$ agarose gels and then visualized by FluorChem (AlphaInnotech) after staining with ethidium bromide. The identity of these PCR products was confirmed by DNA sequencing.

\subsection{Cloning and Construction of Plasmids}

The full-length SICIPK2 was amplified by PCR with cDNA prepared from flower-derived RNA, KOD plus polymerase (TOYOBO) and gene specific primers of SICIPK2-FL-5 and SICIPK2-3 (Table 1), according to the manufacturer's manuals. After digesting by BamH I and Sal I, the DNA fragment was ligated between BamH I and Sal I of pMal-c, for expression of maltose-binding fusion protein (New England Biolabs, Massachusetts, USA). DNA fragments of SICBL1, 2, 10, SIERF7, SICBF1, SIABF4, SIAREB1 and SIPP2C were amplified by PCR with KOD plus DNA polymerase and sets of gene specific primers (Table 1). PCR products of SICBLS1, 2, 10, SIERF7, SICBF1, SIABF4 and SIAREB were digested by BamH I, and then inserted between BamH I and Sma I of pGEX4T-1 (Novagen, Darmstadt, Germany) in ligation. A PCR product of SIPP2C was cloned in PCR TOPO cloning kit (Invitrogen) and then subcloned into pGEX4T-1.
Sequences of the cloned genes were confirmed by sequencing with an ABI Prism 310 DNA sequencer and Big Dye Terminator Cycle Sequencing Kit ver.1.1.

\subsection{Expression and Purification of GST- and MBP-Fused Proteins}

MBP-SICIPK2 was purified with an amylose resin column according to the manufacturer's (New England Biolabs) instruction manual. MBP-SICIPK2 was expressed in E. coli BL21DE3 (Stratagene, La Jolla, CA, USA) containing pMal-c-SICIPK2 by adding isopropyl b-D-1-thiogalactopyranoside (IPTG) at a final concentration of $0.1 \mathrm{mM}$ when the $\mathrm{OD}_{600}$ of $E$. coli culture reached 0.5 . After harvesting of $E$. coli cells $(250 \mathrm{~mL}, 2 \times \mathrm{YT}$ medium) by centrifugation at $10,000 \times \mathrm{g}$ for $15 \mathrm{~min}$, the E. coli pellet was resuspended in lysis buffer, containing $25 \mathrm{mM}$ Tris $\cdot \mathrm{Cl}$ [pH 7.5], $150 \mathrm{mM} \mathrm{NaCl}, 5 \mathrm{mM}$ EDTA, $10 \%$ glycerol, $1 \%$ Triton X-100, $1 \mathrm{mM}$ PMSF, and $0.1 \%$ b-mercaptoethanol, and then the cells were disrupted by sonication. After centrifugation of the mixture at 10,000 $\times \mathrm{g}$ for $15 \mathrm{~min}$ at $4^{\circ} \mathrm{C}$, the resultant supernatant was applied to a column $(1 \mathrm{~cm} \times 2 \mathrm{~cm})$ of amylose resins, and the column was washed with $20 \mathrm{~mL}$ of washing buffer containing $25 \mathrm{mM}$ Tris-Cl [pH 7.4], $1 \mathrm{M} \mathrm{NaCl}, 0.5 \mathrm{mM}$ EDTA, $0.1 \%$ Triton X-100, and $0.1 \%$ b-mercaptoethanol, then washed with elution buffer $(2 \mathrm{~mL})$, which is washing buffer supplemented with $10 \mathrm{mM}$ maltose. The elutant was dialyzed overnight at $4^{\circ} \mathrm{C}$ in $1 \times$ TBS $(25 \mathrm{mM}$ Tris. HCl [pH 7.4], $150 \mathrm{mM} \mathrm{NaCl)} \mathrm{containing} 0.5 \mathrm{mM}$ EDTA, $0.1 \%$ Triton X-100, 0.1\% b-mercaptoethanol, and $30 \%$ glycerol and then stored at $-40^{\circ} \mathrm{C}$.

GST-fusion proteins were purified from E. coli extracts with glutathione CL4B beads, according to the manufacturer's (GE Healthcare Life Sciences) instruction manual. Growth of E. coli cells containing pGEX plasmids, protein induction by IPTG and preparation of $10,000 \times \mathrm{g}$ supernatnat were performed as for MBP-fusion protein. The resultant supernatant was incubated with $200 \mathrm{~mL}$ of 
Table 1. Primers used for RT-PCR analysis and construction of expression plasmids.

\begin{tabular}{|c|c|c|}
\hline Gene & Accession number and/or KTU contig & Primer set \\
\hline SICIPK2-FL-5 & AB674507 & 5-TGGGGATCCGAATTGTTGGTTCAAATGGAG-3 \\
\hline SICIPK2-5 & & 5-AAAGGATCCGAGTCTAGAAACACGGTAACC-3 \\
\hline SICIPK2-3 & & 5-CATGTCGACACGATTAGCTGGCTGCTCGCC-3 \\
\hline SICIPK1-5 & KTU3 contig28893 & 5-GATGGATCCTTGGAAAGTGATGATCACGTC-3 \\
\hline SICIPK1-3 & & 5-AATGTCGACGCTATCACAACAAACAGTAGG-3 \\
\hline SICIPK8-5 & KTU3 contig25787 & 5-GAAGGATCCAACGAGCAATGTGACAATGCG-3 \\
\hline SICIPK8-3 & & 5-AAAGTCGACTCTCTTTCTACTCCTTGCTTT-3 \\
\hline SISOS2-5 & AJ717348 & 5-TACGGATCCGTTAAAGCTAAAGCAGATGAA-3 \\
\hline SISOS2-3 & & 5-ATCTGTCGACGCGAGTCCTTGTCCTAAGCA-3 \\
\hline SICBL1-5 & AB675687 & 5-CTCGGATCCATGGGCTGCTTTAATTCTAAG-3 \\
\hline SICBL1-3 & & 5-ATGGTCGACATTTTCCTAGTGTTATGTAGC-3 \\
\hline SICBL2-5 & AB675688 & 5-GACGGATCCATGCTGCAGTGCTTAGGTTCT-3 \\
\hline SICBL2-3 & & 5-ATGGTCGACTATGTTCATGGAATCATAATT-3 \\
\hline SICBL10-5 & AB675689 & 5-TACGGATCCATGGATTCCACTCGAAATTCT-3 \\
\hline SICBL10-3 & & 5-TTAGTCGACAACTTGTCACAACAAATGGAT-3 \\
\hline SISOS3-5 & AB675686 & 5-СССТСТСТТСТАAАGAАTATGАСССТТСС-3 \\
\hline SISOS3-3 & & 5-GCTAAAAGCTTGAGTCTAGACTTCCG-3 \\
\hline SISOS1-5 & AB675690 & 5-GCGGGATCCCTATCAGGCAGGGGTCTCCAG-3 \\
\hline SISOS1-3 & & 5-CTGCCATGGATGTTTTATCTTCCTCTGCG-3 \\
\hline SlUbi-5 & ВТ012698 & 5-ACGTGGATCCATGCAAATCTTTGTGAAGAC-3 \\
\hline SlUbi-3 & & 5-AAAGTCGACTAACCACCACGGAGACGGAGG-3 \\
\hline SIERF7-5 & AB675691 & 5-TCTGGATCCATGCGGAGAAGCAGAGCAGCC-3 \\
\hline SIERF7-3 & & 5-ATTGTCGACAAGACATAGTGCTGTGCAGTG-3 \\
\hline SICBL1-5 & AY497899 & 5-TCAGGATCCATGAATATCTTTGAAACCTAT-3 \\
\hline SICBF1-3 & & 5-TTAGATAGAATAATTCCATAAAGTTATACT -3 \\
\hline SIABF4-5 & AB675692 & 5-TTTGTCGACACCATGGGATCCTACCTGAAC-3 \\
\hline SIABF4-3 & & 5- CTCGTCGACTGACAACCAAGGTCCCGTCAC-3 \\
\hline SIAREB-5 & AY530758 & 5-ATGGGATCCATGGGGAGTAATTATCATTTCAAG-3 \\
\hline SIAREB-3 & & 5-CTTAAGCTTCCATGGACCAGTTTGTGTCCGTCT-3 \\
\hline SIPP2C-5 & AB490813 & 5-ATCTAGAAATGGAAGAGATGTCTCCAGCTGTTG-3 \\
\hline SIPP2C-3 & & 5-TCAGCTCTAACTTTTGCTTTTGAACTTCСT-3 \\
\hline
\end{tabular}


$50 \%$ glutathione CL4B beads slurry $(\mathrm{v} / \mathrm{v})$ by rotating at $4^{\circ} \mathrm{C}$ for 2 hours. After centrifugation at $3000 \mathrm{rpm}$ for 1 $\min$ at $4^{\circ} \mathrm{C}$, the supernatants were discarded. The resultant GST-fusion protein bound beads were washed 5 times by centrifugation and adding washing buffer mention above. The purified GST-fusion proteins bound on glutathione bead were added with $30 \%$ glycerol at final concentration and then stored at $-30^{\circ} \mathrm{C}$.

\subsection{Immunoblot with Anti-CIPK Antibody, Anti-Tomato CBL4/SOS3 Antibody}

After SDS-PAGE, separated polypeptides were electroblotted onto a PVDF membrane at $10 \mathrm{~V} \mathrm{~cm}^{-1}$ for $90 \mathrm{~min}$ in $25 \mathrm{mM}$ Tris-base containing $0.05 \%$ SDS and $20 \%$ methanol) and then the PVDF membrane (Millipore) was blocked by incubating in $1 \times$ TBS containing $3 \%$ skim milk (TBS-milk) for 60min. The PVDF membrane was incubated with anti-CIPK antibody (one to 2000 dilution [v/v]) or anti-tomato-SOS3 antibody (one to 2000 dilution [v/v]) [16] in TBS-milk containing 0.05\% Tween 20 at room temperature for 2 hours. Subsequently, the PVDF membrane was incubated at room temperature for $60 \mathrm{~min}$ in TBS containing horseradish peroxidase (HRP) conjugated protein G (GE Healthcare Bio-Sciences, Piscataway, NJ, USA) (one to 10,000 dilution $[\mathrm{v} / \mathrm{v}]$ ), and $0.05 \%$ Tween 20. The immunodecorated protein bands were visualized by an ECL Plus kit (GE Healthcare Bio-Sciences) and FluorChem Imaging Analyzer.

\subsection{Preparation of Extracts from Plant Tissues}

Frozen tissues ( $2 \mathrm{~g}$ ) were homogenized on an ice-cooled motor in $6 \mathrm{~mL}$ of lysis buffer containing $20 \mathrm{mM} 3-(\mathrm{N}-$ morpholino)-propanesulfonic acid (MOPS, $50 \mathrm{mM} \mathrm{Na} 2$-bglycerophosphate [pH 7.6], $10 \mathrm{mM}$ EDTA, 5 mM EGTA, $30 \mathrm{mM} \mathrm{NaF}, 5 \mathrm{mM} \mathrm{Na} \mathrm{VO}_{4}$, $5 \%$ glycerol, $0.1 \%$ b-mercaptoethanol, $1 \mathrm{mM}$ PMSF, $5 \mathrm{mM} n$-aminocaproic acid, $1 \mathrm{mM}$ benzamidine, $1 \mathrm{mM}$ Na-bisulfite, and $1 \mu \mathrm{g} / \mathrm{mL}$ leupeptin, with or without $1 \%$ Triton X-100. The resultant homogenates were centrifuged at $10,000 \times \mathrm{g}$ for 20 $\min$ at $4^{\circ} \mathrm{C} .100,000 \times$ g supernatants (soluble fractions) and $100,000 \times \mathrm{g}$ pellets (microsomal fractions) were prepared by centrifugation at $100,000 \times \mathrm{g}$ for $60 \mathrm{~min}$ at $4^{\circ} \mathrm{C}$ (P65A rotor and 55P-7 ultra-centrifugator, Hitachi Inc., Tokyo, Japan) with 10,000 $\times$ g supernatant fraction (without Triton X-100) described above. Protein concentrations in samples were measured by a Bio Rad protein assay kit (BioRad, Hercules, CA, USA) with bovine serum albumin (BSA) as a standard.

\subsection{In Vitro Binding Assay}

Ten micrograms each of GST and GST-fusion proteins bound on $20 \mathrm{~mL}$ of glutathione Sepharose beads (50\% [v/v] slurry) in $1.5 \mathrm{~mL}$ of binding buffer containing $1 \times$ TBS, $0.1 \%$ Triton X-100, and 0.1\% b-mercaptoethanol, were added to $10 \mu \mathrm{g}$ of purified MBP-SICIPK2. After rotation at $4^{\circ} \mathrm{C}$ for 2 hours, the GST-fusion protein-immobilized beads were briefly centrifuged at $5000 \times \mathrm{g}$ for $10 \mathrm{~s}$ and then washed 5 times with $1 \mathrm{~mL}$ of washing buffer containing $1 \times$ TBS, $0.5 \mathrm{M} \mathrm{NaCl}, 0.1 \%$ Triton X-100, and $0.1 \% \mathrm{~b}$-mercaptoethanol. After these washings, the precipitates with beads were separated by SDS-PAGE and the separated polypeptides were visualized by staining with Coomassie Brilliant Blue R250.

\subsection{Construction of Green Fluorescence Protein Plasmids and Fluorescence Microscopy}

A multicloning site containing $\mathrm{Bgl}$ II and Sal I was inserted at 3'-end of GFP(S65T) gene of an original GFP expression vector [17]. SICIPK2 PCR product was digested with BamH I and Sal I as described in the construction of pGEX-SICIPK2, and then was ligated into the Bgl II and Sal I site of pCaM35S-pro-GFP(S65T) (This study). The resultant pCaM35S-pro-GFP(S65T)-SICIPK2 plasmid and the pCaM-pro-GFP(S65T) empty vector were introduced into onion epidermal cells with a particle bombardment (PDS-100/He, BioRad Ltd.) using 1.0- or 1.6- $\mu$ m gold particles at 1100 psi. After incubation in Murasige-Skoog medium containing $3 \%$ sucrose for 24 hours, the onion cells were subjected to fluorescence microscopy analysis (ECLIPSE 80i, Nikon Ltd., Tokyo, Japan) with a filter system of BP480nm/DM550nm/LP510nm.

\section{Results}

\subsection{A Flower Specific Expression of a Tomato CIPK Homolog}

A phylogenic tree indicates that the tomato CIPKs and the corresponding Arabidopsis CIPKs formed a distant group from other SNF1-related kinases including SnRK I and SnRK II subfamilies and CDPK among the CDPKSNF1 superfamily, as expected (Figure 1(b)). A significant signal of SICIPK2 was detected specifically in flower at 25 cycles, and the expression level of SICIPK2 is the highest in flower than in other organs at 28 or even 31 cycles (Figure 1(a)). In contrast, SICIPK1 were detected ubiquitously in all organs at 30 and 33 cycles (Figure 1(a)). At 27 cycles, the relative signals of SICIPK8 and SISOS2 were much weaker than those of SICIPK1, while those were expressed in all organs. SISOS1, expression of putative $\mathrm{Na}^{+} / \mathrm{H}^{+}$antiporter, was also detected ubiquitously in all organs.

Next, a phylogenic tree of CBLs indicates relationships of tomato CBLs with the corresponding Arabidopsis CBLs by using tomato calmodulin (LeCaM) and AtCaM1 as outgroup (Figure 1(b)). Among CBL homologs, the expres- 
sion of SISOS3 were significant in leaf but not or marginal in flower at 27 and 30 cycles. The signals of SICBL1 and SICBL2 in flower were weaker than those in leaf at 27 cycles, but the difference of expression of SICBL1 and SlCBL1 between leaf and flower was not apparent at 30 cycles. The signals of SICBL10 showed no or marginal difference in transcript level between leaf and flower.

\subsection{Localization of CIPK-Related Proteins and CBL-Related Proteins in Vaious Organs of Tomato}

It was confirmed that the anti-cowpea CIPK antibody cross-reacts with MBP-SICIPK2 but not with MBP only (Figure 3(a)). The anti-CIPK antibody can cross-react broadly with with CIPK-related proteins in higher plants, tomato, soybean, cowpea and Arabidopsis thaliana [16].

Immunoblot by the anti-CIPK antibody showed a significant signal in flower with a relative molecular mass of $55 \mathrm{kDa}$ (Figure 3(b)) in the presence or the absence of Triton X-100. Weak signals were also detected in young seedling and leaf in the presence of Triton X-100 at about $55 \mathrm{kDa}$ but no or marginal in the absence of Triton $\mathrm{X}-100$. Immuno-reactive signals in root, stem and fruit were only very weak or faint. Next, the tissue specific profiles of the CIPK-related proteins in flower was determined by immunoblot using extracts, which were prepared by $10,000 \times$ g centrifugation with homogenates from various tissues of tomato flower and plant in the presence of Triton X-100 (Figure 4(a)). The immunoreactive signals at $55-\mathrm{kDa}$ were significant in stamen, and very low in petal, ovary and young fruit, but no or scarcely in leaf and petal.

To examine the intracellular localization of the flowerspecific CIPK-related polypeptide, ultracentrifugation was carried out with the $5000 \times \mathrm{g}$ supernatant fractions from homogenates of leaf and flower without Triton $\mathrm{X}-100$. A significant signal was detected in 50,000 $\times \mathrm{g}$ supernatant and $100,000 \times \mathrm{g}$ pellet in flower but not in $100,000 \times \mathrm{g}$ in flower. In contrast, there was no or marginal immunoreactive signal in samples of leaf (Figure 4(b), right). Several Arabidopsis CBLs are covalently bound to a myristoyl moiety on glycine at the N-terminus leading to recruitment of the CBL-CIPK complex on the microsomes [18]. Immunoblot revealed the SOS3-reated peptide at about $28 \mathrm{kDa}$ in $100,000 \times$ g pellets but not in $100,000 \times \mathrm{g}$ supernatants of leaf extracts (Figure 4(c), left). In contrast, there was no or marginal immunoreactive signals both in $100,000 \times \mathrm{g}$ super4C, right).

\subsection{Interaction of SLCIPK2 with CBL Homologs and Transcriptional Factors}

The deduced aimino acid sequence of SICIPK2 indicates the presence of CBL-interacting domain called "NAF/ FISL motif" at 312 - 333 aa in the C-terminus [10]. Therefore, we used in vitro binding assay with a MBP-SICIPK2 and GST-SICBLs to examine whether SICIPK2 associates with SICBL1, 2 and 10, because it have been reported that the CBLs have different preference for potential counterparts of CIPKs [19]. In vitro binding assay of MBP-SICIPK2 was performed using GST and GST-SICBL1, 2 and 10. MBP-SICIPK1 was detected in pull-downed samples with GST-SICBL1, 2 and 10 but not with GST (Figure 5(a)). Interestingly, the presence or absence of $\mathrm{Ca}^{2+}$ in the binding buffer had marginal or no effect on the interaction of MBP-SICIPK2 with GSTSICBL1, 2 nor 10 (data not shown), although the predicted amino acid sequences of SICBLs contain typical EF-hand motives that potentially bind with $\mathrm{Ca}^{2+}$. The $\mathrm{Ca}^{2+}$-dependent association of CIPKs with CBLs and the resultant activation is dependent on preferential combinations of

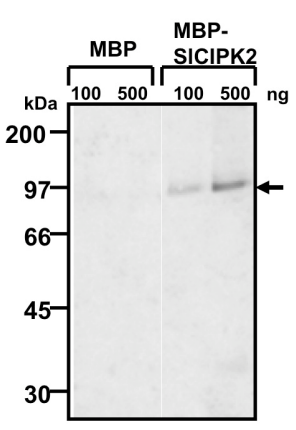

(a)

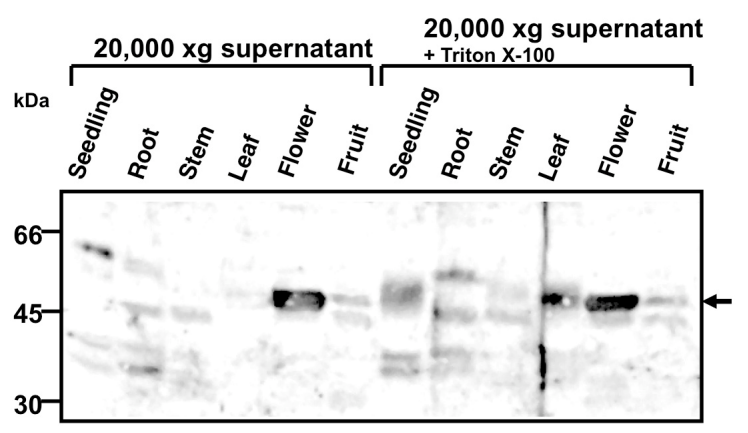

(b)

Figure 3. Detection of immunologically CIPK-related proteins in various organs of tomato. Recombinant SICIK2 (a) and protein extracts prepared from seedling, root, stem leaf, flower and fruit (b) were analyzed by immunoblot with anti-CIPK specific antibody and ECL kit. Recombinant protein of MBP-SICIPK2 and MBP were subjected to immunoblot by the antibody using indicated amounts of proteins. The $10,000 \times \mathrm{g}$ soluble fractions were prepared from crude extracts of leaves of each plants in the presence (right) or the absence (left) of $1 \%$ Triton $\mathrm{X}-100$. Each $50 \mu \mathrm{g}$ protein extracts per lane was subjected to SDS-PAGE (10\% acrylamide gels) and electroblotted to PVDF membrane. 


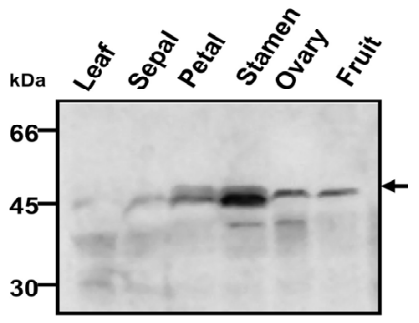

(a)

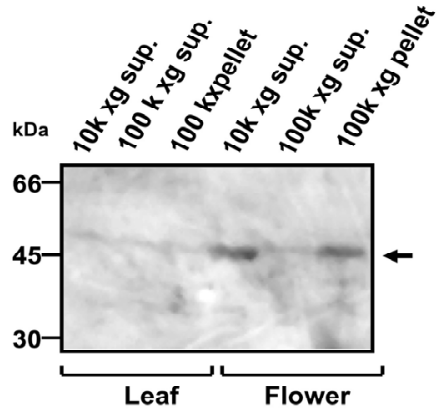

(b)

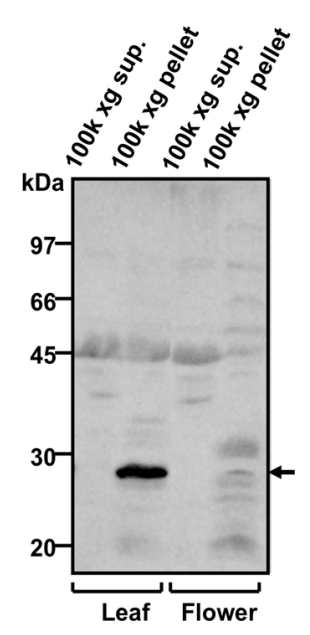

(c)

Figure 4. Immunoblot of CIPK-related polypeptides and SOS3-related polypeptides in leaf and flower of tomato plant. Immunoblot using solubilized protein extracts prepared from leaf, sepal, petal, stamen, ovule and young fruit of tomato plant was carried out by anti-CIPK antibody (a). The soluble fraction and microsomal fraction prepared from leaf and flower were subjected to immunoblot by anti-CIPK antibody (b) and anti-SISOS3 antibody (c). The $10,000 \times \mathrm{g}$ soluble fractions were prepared with lysis buffer containing 1\% Triton X-100 from crude extracts of leaf and flower of 6-8-week-old tomato plant. Fractionation of $100,000 \times \mathrm{g}$ soluble fraction and $100,000 \times \mathrm{g}$ pellets was carried out at $100,000 \times \mathrm{g}$ for $60 \mathrm{~min}$ at $4{ }^{\circ} \mathrm{C}$ after preparation of $10,000 \times \mathrm{g}$ supernatants in the absence of Triton $\mathrm{X}-100$. Each $50 \mu \mathrm{g}$ protein per lane was subjected to SDS-PAGE (10\% or $12.5 \%$ acrylamide gels) and immunoblot by anti-CIPK serum and anti-tomato CBL serum, respectively.

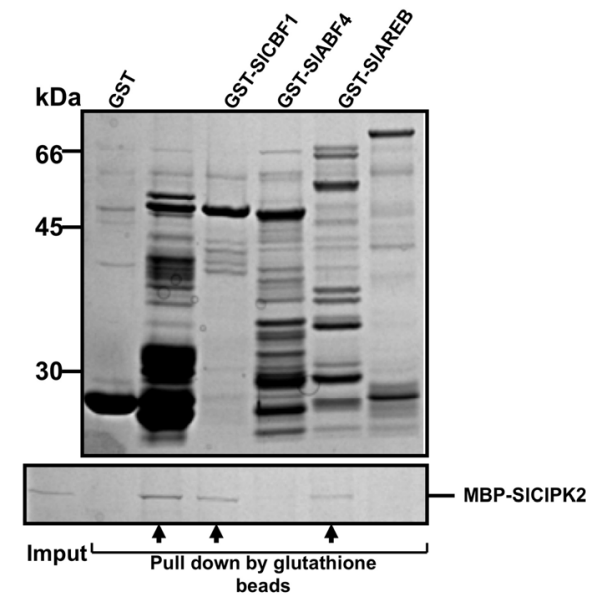

(a)

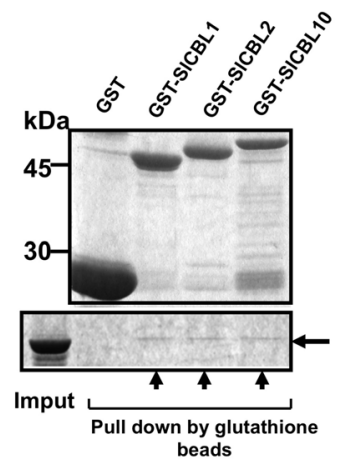

(b)

Figure 5. Interaction of SICIPK2 with tomato CBL homologs and transcription factors. Pull-down assay with glutathionesepharose beads was carried out with MBP-SICIPK2, GST, and GST-tomato CBL1, CBL2 and CBL10 in the presence of 1 $\mathrm{mM} \mathrm{CaCl}_{2}$ (a). Recombinant proteins of tomato stress-responsive transcription factors and SIPP2C were subjected to in vitro binding assay of SICIPK2 (b). Signals of MBP-SICIPK2 co-precipitated with GST-fusion proteins are indicated by an arrow.

between individual CBLs and CIPKs [19]. For example, the $\mathrm{Ca}^{2+}$-independent interaction was observed between AtCBL4/SOS3 and AtCIPK24/SOS2 [15], as is the interaction between SICIPK2 and SICBL1, 2 and 10.

Recent studies revealed that AREB/ABF- and AP2/ERFtype transcription factors are implicated in $\mathrm{ABA}$ and osmotic stress signaling [20,21]. Stress-activated SnRKs potentially phosphorylate those transcription factors [2226]. Therefore, in vitro interaction assay between SICIPK2 and the tomato transcription factors, ERF7, CBF1, ABF4 and AREB1 was conducted. Furthermore, protein phosphatases type-2C (PP2C) appeaed to be involved in downregulation and dephosphorylation of CIPKs and SnRK2s and interacting with those specific CIPKs and SnRK2s $[20,21]$. Therefore, GST-SIPP2C was also subjected to the in vitro binding assay with SICIPK2. MBP-SICIPK1 was detected in pull-downed samples with GST-SIERF7, CBF1 and AREB1but not with GST nor GST-ABF4 
(Figure 5(b)). There was no signal of MBP-SICIPK2 in the pull-downed sample with GST-SIPP2C.

\subsection{Subcellular Localization of GFP-Fused SLCIPK2}

We investigated the distribution of GFP fluorescence of GFP-fused SICIPK2 after transient expression into onion epidermal cells (Figure 6(a)). In these experiments, we observed a dispursed distribution of GFP-SICIPK2 in the cytosol and a significant signal in whole nucleous. The pattern of subcellular distribution of GFP-SICIPK2 distribution resembled to those of GFP alone, while the fluorescneces of GFP-SICIPK were reltively weaker than those of GFP alone.

\section{Discussion}

The tomato and Arabidopsis CIPKs formed a monophyletic cluster separated from other clusters of SnRK1 groupe, LeSNF1 and yeast SNF1 [27], and of SnRK2 groupe, SISnRK2C and AtSnRK2.8, while NtCDPK1 is located as outgroup $[12,28]$. SlCIPK2 has the highest similarity (62\%) in amino acid sequence to that of AtCIPK2, and SICIPK1, SICIPK8 and SISOS2 have the highest similarities (66, 70 and 72\%) in amino acid sequences to those of AtCIPK1, AtCIPK8 and AtCIPK24/ SOS2, respectively. SICIPK2 and AtCIPK2 are placed in the subclass including AtCIPK15/PKS3, which is involved in $\mathrm{Ca}^{2+}$-regulated ABA perception [29], while AtCIPK24/ SOS2, which is involved in $\mathrm{Na}^{+}$excretion [30], and AtCIPK23, which is involved in regulation of $\mathrm{K}^{+}$channels [14], belong to another subclass of CIPK family includeing a set of CIPK homologs involving ion regulation of ion transport. In contrast to the expression profiles of SICIPK2 mRNA (Figure 1), other tomato CIPK homologs are broadly expressed in various tissues at relatively low levels, compared with that of SICIPK2 in

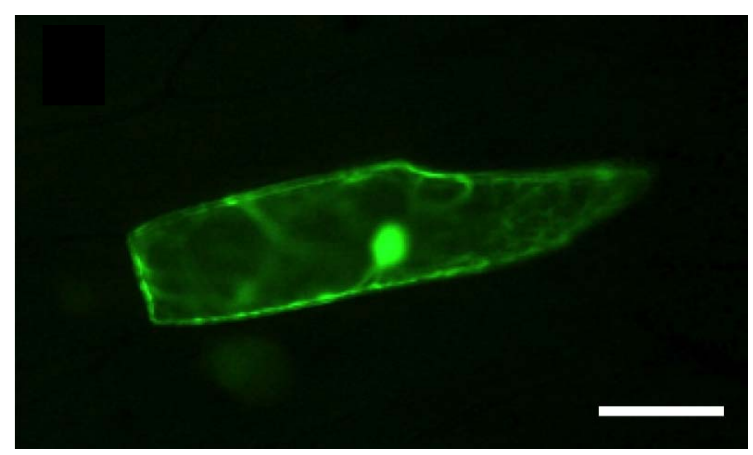

(a) flower. The high expression level of SICIPK2 in flower (Figure 1(b)) is consistent with the observation that significant immnoreactive signal of the CIPK-related polypeptide in flower and stamen (Figure 3(b)). It is well known that osmolyres, proline and sucrose, are highly accumulated in pollen and that severe drought and heat stress on pollen development have critical effects on the efficiency of the germination and fertilization. It is conceivable that SICIPK2 functions on regulation of osmolyte-uptake mechanism in pollen. When expression patterns of SICIPK1, 2 and 8 and SISOS2 were examined using whole plant treated by various stresses, drought, $\mathrm{NaCl}, \mathrm{ABA}$, and chilling stress, no or marginal alteration in expression levels of those homologs were detected (data not shown).

SISOS3 among tomato CBLs is preferentially expressed in leave, but less or scarcely in flower, while SICBL10 was expressed in both leave and flower at the same levels. In vitro binding assay suggests that SICBL1, 2 and 10 have potential to interact with SICIPK2. In Arabidopsis, SOS3 appeared to interact with SOS2, which is involved in $\mathrm{Na}^{+}$-extrusion and salt tolerance of root. In tomato, SISOS3 is induced under salt stress (Takahashi, Yuasa and Iwaya-Inoue, in preparation). Therefore, our observations and specific interaction between SOS3 and SOS2, previously reported, suggest that those CBL10 and in part, CBL1 and 2, possibly function at upstream of SICIPK2 and that SISOS3 may be involved in salt stress signaling but not in flower specific functions.

Results of RT-PCR and immunoblot indicate that the CIPK-related protein and the SOS3-related protein are expressed in flower and leaf alternatively, and that both proteins are preferentially localized in the microsomal fraction but not in the soluble fraction. The N-terminus of SISOS3 contains a typical consensus motif, " $\mathrm{H}_{2} \mathrm{~N}-\mathrm{M}$ G-C-F-P-S-", recognized as " $\mathrm{H}_{2} \mathrm{~N}-\mathrm{M}-\mathrm{G}-\mathrm{X}-\mathrm{X}-\mathrm{X}-(\mathrm{S} / \mathrm{T})$-" for myristoylation, suggesting that SISOS3 is preferentially

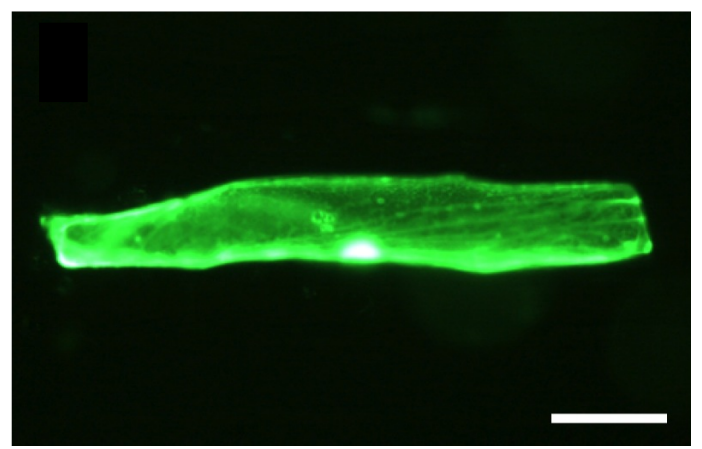

(b)

Figure 6. Green fluorescence proteins in onion epidermal cells introduced with plasmids expressing GFP-fused SICIPK2 (a) and GFP alone (b). Onion epidermal cells were incubated on Murasige-Skoog medium containing $3 \%$ sucrose for 24 hours after gold particles coated with pCaM35S-pro-GFP(S65T)-fused SICIPK2 and pCaM35S-pro-GFP(S65T) by particle gun. GFP expressed in onion epidermal cells was observed on a fluorescence microscopy. Bars indicated are $50 \mu \mathrm{m}$. 
localized on the plasma membrane as SOS3 in Arabidopsis [31]. The SICIPK2 has no apparent membrane associating domain in itself, while the flower specific CIPK was detected in the membrane fraction (Figure 4). Because that SICBLl has the myristoyl motif " $\mathrm{H}_{2} \mathrm{~N}-\mathrm{M}-\mathrm{G}-\mathrm{C}$ F-N-S-" on its N-terminus, it is possible that SlCBL1 and/ or other lipid-modified CBL molecules recruit SICIPK2 onto the membrane. In contrast to the result from subcellular fructionation (Figures 3(b) and 4(b)), the fluorescence of GFP-SICIPK2 expressed in onion epidermis cells were detected in the cytosol and nucleous (Figure 6(a)). It was reported that distinct targeting signals located in the N-terminal domain of CBLs determine the spatially discrete localization of CBL/CIPK complexes via specific interaction set between CBLs and CIPKs [32]. Confocul microspcopic observation with coexpression of GFP-CIPKs and lipid-modified CBLs displayed significant fluorescence signals on the plasma membrane and/or the tonolplast, while expression of GFP-CIPKs alone showed the fluorescence signals in the cytosol and nucleous. As consistent to the previous stuides on CBL-CIPK, the subcellular fractionation and the fluorescence microscpic observation in the present study suggest that the membrane association of SICIPK2 requires interaction with a specific CBLs possessing its membrane targeting signal.

There has been growing evidence that SnRK2s and SnRK3s participate coordinately in stress-stimulated gene expression by phosphorylating different sets of transcription factors in response to environmental stresses and hormonal signals, while previous studies have been focusing on regulation of ion transport on plasma membrane and in vitro binding assay showed that SICIPK2 can associate with SIERF7, SICBF1 and SIAREB. Recently, interaction of ABA signal-related PP2Cs, ABI1/ABI2 with SnRK2.6/AtSRK2E and SOS2 has been demonstrated [33,34]. While in vitro pull-down assay failed to detect association of SICIPK2 with SIPP2C (Figure 5(b)), a possibility is not excluded that SICIPK2 is regulated by other PP2C homologs.

The present study revealed unique features of SICIPK2: 1) SICIPK2 mRNA is expressed specifically in flower; 2 ) The CIPK-related protein is detected at a significant level in stamen; 3) The flower specific CIPK tightly associates with microsomal fraction of flower; 4) SICIPK2 can interact to stress-responsive transcription factors. These observations suggest that SICIPK is possibly involved in floral organ specific functions rather than salt stress signaling mediated by SISOS3. It is necessary for clarifying the intracellular localization and the tissue specific expression of SICIPK2 to perform immunofluorscence microscopic analysis and in-situ hybridization. While interaction of SICIPK2 with CBL1, 2 and 10 was detected in in vitro binding assay, it is conceivable that the specific
CBLs are required for association and function of SICIPK2 on the membrane. It was reported that accumulation of proline and sucrose in pollen at high concentration is required for the development, maturation and desiccation. The osmolyte uptake mechanisms in pollen may be accounted for stress signaling cascades utilizing flowerspecific SICIPK2 and CBLs which regulate solute transport and water permieability ofstamen and pollen cells. It remains to be clarified whether SICIPK2 phosphorylates SIERF7, SICBF1 and SIAREB, leading to activation of stress-inducible genes in flower, or whether it is involved in the direct regulation of transporters on vacuole and plasma membrane in floral tissues by phosphorylation along developmental process of pollen.

Increasing evidence suggests that CBL-CIPK complexes phosphorylate and activate various ion transporters, such as $\mathrm{Na}^{+} / \mathrm{H}^{+}$exchangers and $\mathrm{K}^{+}$channels $[14,35]$, in vegetative organ such as root, stem and leave, whereas there were few reports about flower specific CIPK nor SnRK2. Therefore, identification of the activator or signaling regulated regulating SICIPK2 in floral organ is important for improvement of stress tolerance of reproductive organ.

\section{Acknowledgements}

This work was supported in part by Grant-in-Aids from the Ministry of Education, Sports, Culture, Science and Technology of Japan, No. 19380022 (to T. Y.) and Salt Science Research Foundation, No. 0827 (to T.Y.).

\section{REFERENCES}

[1] N. Yabe, T. Takahashi and Y. Komeda, "Analysis of Tissue-Specific Expression of Arabidopsis thaliana HSP90Family Gene HSP81,” Plant and Cell Physiology, Vol. 35, No. 8, 1994, pp. 1207-1219.

[2] C. Prasinos, K. Krampis, D. Samakovli and P. Hatzopoulos, "Tight Regulation of Expression of Two Arabidopsis cytosolic Hsp90 Genes during Embryo Development,” Journal of Experimental Botany, Vol. 56, No. 412, 2005, pp. 633-644. doi:10.1093/jxb/eri035

[3] R. Schwacke, S. Grallath, K. E. Breitkreuz, E. Stransky, H. Stransky, W. B. Frommer and D. Rentsch, "LeProT1, a Transporter for Proline, Glycine Betaine, and GammaAmino Butyric Acid in Tomato Pollen,” The Plant Cell, Vol. 11, No. 3, 1999, pp. 377-392. doi:10.1105/tpc.11.3.377

[4] K. Shinozaki and K. Yamaguchi-Shinozaki, "Molecular Responses to Dehydration and Low Temperature: Differences and Cross-Talk between Two Stress Signaling Pathways," Current Opinion in Plant Biology, Vol. 3, No. 3, 2000, pp. 217-223. doi:10.1016/S1369-5266(00)80068-0

[5] J. L. Brewster, T. de Valoir, N. D. Dwyer, E. Winter and M. C. Gustin, "An Osmosensing Signal Transduction Pathway in Yeast,” Science, Vol. 259, No. 5102, 1993, pp. 


\section{0-1763. doi:10.1126/science.7681220}

[6] K. Ichimura, K. Shinozaki, G. Tena, J. Sheen, C. V. Giordano, A. Acevedo, A. L. Scopel and C. L. Ballare (MAPK Group), "Mitogen-Activated Protein Kinase Cascades in Plants: A New Nomenclature," Trends in Plant Science, Vol. 7, No. 7, 2002, pp. 301-308. doi:10.1016/S1360-1385(02)02302-6

[7] J. Li, X. Q. Wang, M. B. Watson and S. M. Assmann, "Regulation of Abscisic Acid-Induced Stomatal Closure and Anion Channels by Guard Cell AAPK Kinase," Science, Vol. 287, No. 5451, 2000, pp. 300-303. doi:10.1126/science.287.5451.300

[8] T. Umezawa, R. Yoshida, K. Maruyama, K. YamaguchiShinozaki and K. Shinozaki, "SRK2C, a SNF1-Related Protein Kinase 2, Improves Drought Tolerance by Controlling Stress-Responsive Gene Expression in Arabidopsis thaliana," Proceedings of the National Academy of Sciences of the United States of America, Vol. 101, No. 49, 2004, pp. 17306-17311. doi:10.1073/pnas.0407758101

[9] T. Yuasa, Y. Tomikubo, T. Yamauchi, A. Inoue and M. Iwaya-Inoue, "Environmental Stresses Activate a Tomato SNF1-Related Protein Kinase 2 Homolog, SISnRK2C,” Plant Biotechnology, Vol. 24, No. 4, 2007, pp. 401-408. doi:10.5511/plantbiotechnology.24.401

[10] V. Albrecht, O. Ritz, S. Linder, K. Harter and J. Kudla, "The NAF Domain Defines a Novel Protein-Protein Interaction Module Conserved in $\mathrm{Ca}^{2+}$-Regulated Kinases,” The EMBO Journal, Vol. 20, No. 5, 2001, pp. 1051-1063. doi:10.1093/emboj/20.5.1051

[11] Y. Guo, U. Halfter, M. Ishitani and J.-K. Zhu, "Molecular Characterization of Functional Domains in the Protein Kinase SOS2 That Is Required for Plant Salt Tolerance,” The Plant Cell, Vol. 13, No. 6, 2001, pp. 1383-1400. doi:10.1105/tpc.13.6.1383

[12] E. M. Hrabak, C. W. Chan, M. Gribskov, J. F. Harper, J. H. Choi, N. Halford, J. Kudla, S. Luan, H. G. Nimmo, M. R. Sussman, M. Thomas, K. Walker-Simmons, J. K. Zhu and A. C. Harmon, "The Arabidopsis CDPK-SnRK Superfamily of Protein Kinases,” Plant Physiology, Vol. 132, No. 2, 2003, pp. 666-680.

doi:10.1104/pp.102.011999

[13] V. Albrecht, S. Weinl, D. Blazevic, C. D’Angelo, O. Batistic, U. Kolukisaoglu, R. Bock, B. Schulz, K. Harter and J. Kudla, "The Calcium Sensor CBL1 Integrates Plant Responses to Abiotic Stresses,” Plant Journal, Vol. 36, No. 4, 2003, pp. 457-470. doi:10.1046/j.1365-313X.2003.01892.x

[14] J. Xu, H. D. Li, L. Q. Chen, Y. Wang, L. L. Liu, L. He and W. H. Wu, "A Protein Kinase, Interacting with Two Calcineurin B-Like Proteins, Regulates $\mathrm{K}^{+}$Transporter AKT1 in Arabidopsis,” Cell, Vol. 125, No. 7, 2006, pp. 1347-1360. doi:10.1016/j.cell.2006.06.011

[15] U. Halfter, M. Ishitani and J.-K. Zhu, "The Arabidopsis SOS2 Protein Kinase Physically Interacts with and Is Activated by the Calcium-Binding Protein SOS3," Proceedings of the National Academy of Sciences of the United States of America, Vol. 97, No. 7, 2000, pp. 3735-3740.

\section{doi:10.1073/pnas.97.7.3735}

[16] M. Imamura, T. Yuasa, T. Takahashi, N. Nakamura, M. P. S. H. Nang, S.-H. Zheng, K. Shimazaki and M. Iwaya-Inoue, "Isolation and Characterization of a cDNA Coding Cowpea (Vigna unguiculata (L.) Walp.) Calcineurin B-Like Protein Interacting Protein Kinase, VuCIPK1,” Plant Biotechnology, Vol. 25, No. 4, 2008, pp. 437-445.

[17] Y. Niwa, T. Hirano, K. Yoshimoto, M. Shimizu and H. Kobayashi, "Non-Invasive Quantitative Detection and Applications of Non-Toxic, S65T-Type Green Fluorescent Protein in Living Plants,” Plant Journal, Vol. 18, No. 4, 1999, pp. 455-463. doi:10.1046/j.1365-313X.1999.00464.x

[18] U. Kolukisaoglu, S. Weinl, D. Blazevic, O. Batistic and J. Kudla, "Calcium Sensors and Their Interacting Protein Kinases: Genomics of the Arabidopsis and Rice CBLCIPK Signaling Networks,” Plant Physiology, Vol.134, No. 1, 2004, pp. 43-58. doi:10.1104/pp.103.033068

[19] O. Batistic and J. Kudla, "Integration and Channeling of Calcium Signaling through the CBL Calcium Sensor/ CIPK Protein Kinase Network,” Planta, Vol. 219, No. 6, 2004, pp. 915-924. doi:10.1007/s00425-004-1333-3

[20] T. Hirayama and K. Shinozaki, "Perception and Transduction of Abscisic Acid Signals: Keys to the Function of the Versatile Plant Hormone ABA,” Trends in Plant Science, Vol. 12, No. 8, 2007, pp. 343-351.

doi:10.1016/j.tplants.2007.06.013

[21] A. Wasilewska, F. Vlad, C. Sirichandra, Y. Redko, F. Jammes, C. Valon, N. F. dit Frey and J. Leung, "An Update on Abscisic Acid Signaling in Plants and More..." Molecular Plant, Vol. 1, No. 2, 2008, pp. 198-217. doi:10.1093/mp/ssm022

[22] Y. Uno, T. Furihata, H. Abe, R. Yoshida, K. Shinozaki, K. Yamaguchi-Shinozaki, “Arabidopsis Basic Leucine Zipper Transcription Factors Involved in an Abscisic AcidDependent Signal Transduction Pathway under Drought and High-Salinity Conditions," Proceedings of the National Academy of Sciences of the United States of America, Vol. 97, No. 21, 2000, pp. 11632-11637. doi:10.1073/pnas.190309197

[23] Y. Kobayashi, M. Murata, H. Minami, S. Yamamoto, Y. Kagaya, T. Hobo, A. Yamamoto and T. Hattori, "Abscisic Acid-Activated SNRK2 Protein Kinases Function in the Gene-Regulation Pathway of ABA Signal Transduction by Phosphorylating ABA Response Element-Binding Factors,” The Plant Journal, Vol. 44, No. 6, 2005, pp. 939949. doi:10.1111/j.1365-313X.2005.02583.X

[24] H. Choi, H. J. Park, J. H. Park, S. Kim, M. Y. Im, H. H. Seo, Y. W. Kim, I. Hwang and S. Y. Kim, “Arabidopsis Calcium-Dependent Protein Kinase AtCPK32 Interacts with ABF4, a Transcriptional Regulator of ABA-Responsive Gene Expression, and Modulates Its Activity,” Plant Physiology, Vol. 139, No. 4, 2005, pp. 1750-1761. doi:10.1104/pp.105.069757

[25] P. Agarwal, R. Arora, S. Ray, A. K. Singh, V. P. Singh, H. Takatsuji, S. Kapoor and A. K. Tyagi, "Genome-Wide Identification of $\mathrm{C} 2 \mathrm{H} 2$ Zinc-Finger Gene Family in Rice and Their Phylogeny and Expression Analysis," Plant 
Molecular Biology, Vol. 65, No. 4, 2007, pp. 467-485. doi:10.1007/s11103-007-9199-y

[26] C. P. Song, M. Agarwal, M. Ohta, Y. Guo, U. Halfter, P. Wang and J.-K. Zhu, "Role of an Arabidopsis AP2/ EREBP-Type Transcriptional Repressor in Abscisic Acid and Drought Stress Responses,” The Plant Cell, Vol. 17, No. 8, 2005, pp. 2384-2396. doi:10.1105/tpc.105.033043

[27] J. L. Celenza and M. Carlson, "Structure and Expression of the SNF1 Gene of Saccharomyces cerevisiae," Molecular and Cellular Biology, Vol. 4, No. 1, 1984, pp. 5460.

[28] S. Ishida, T. Yuasa, M. Nakata and Y. Takahashi, "A Tobacco Calcium-Dependent Protein Kinase, CDPK1, Regulates the Transcription Factor Repression of Shoot Growth in Response to Gibberellins,” The Plant Cell, Vol. 20, No. 12, 2008, pp. 3273-3288.

[29] Y. Guo, L. Xiong, C.-P. Song, D. Gong, U. Halfter and J.-K. Zhu, “A Calcium Sensor and Its Interacting Protein Kinase Are Global Regulators of Abscisic Acid Signaling in Arabidopsis,” Developmental Cell, Vol. 3, No. 2, 2002, pp. 233-244. doi:10.1016/S1534-5807(02)00229-0

[30] Q. S. Qiu, Y. Guo, F. J. Quintero, J. M. Pardo, K. S. Schumaker and J.-K. Zhu, "Regulation of Vacuolar $\mathrm{Na}^{+} / \mathrm{H}^{+}$ Exchange in Arabidopsis thaliana by the Salt-OverlySensitive (SOS) Pathway," Journal of Biological Chemistry, Vol. 279, No. 1, 2004, pp. 207-215. doi:10.1074/jbc.M307982200
[31] M. Ishitani, J. Liu, U. Halfter, C. S. Kim, W. Shi and J.-K. Zhu, "SOS3 Function in Plant Salt Tolerance Requires N-Myristoylation and Calcium Binding,” The Plant Cell, Vol. 12, No. 9, 2000, pp. 1667-1678. doi:10.1105/tpc.12.9.1667

[32] O. Batistic, R. Waadt, L. Steinhorst, K. Held and J. Kudla, "CBL-Mediated Targeting of CIPKs Facilitates the Decoding of Calcium Signals Emanating from Distinct Cellular Stores,” The Plant Journal, Vol. 61, No. 2, 2010, pp. 211-222.

[33] M. Ohta, Y. Guo, U. Halfter and J.-K. Zhu, “A Novel Domain in the Protein Kinase SOS2 Mediates Interaction with the Protein Phosphatase 2C ABI2," Proceedings of the National Academy of Sciences of the United States of America, Vol. 100, No. 20, 2003, pp. 11771-11776. doi:10.1073/pnas.2034853100

[34] R. Yoshida, T. Umezawa, T. Mizoguchi, S. Takahashi, F. Takahashi and K. Shinozaki, "The Regulatory Domain of SRK2E/OST1/SnRK2.6 Interacts with ABI1 and Integrates Abscisic Acid (ABA) and Osmotic Stress Signals," Journal of Biological Chemistry, Vol. 281, No. 8, 2006, pp. 5310-5318. doi:10.1074/jbc.M509820200

[35] J.-K. Zhu, "Regulation of Ion Homeostasis under Salt Stress," Current Opinion in Plant Biology, Vol. 6, No. 5, 2003, pp. 441-445. doi:10.1016/S1369-5266(03)00085-2 